\title{
Making Book History: Engaging Maker Culture and 3D Technologies to Extend Bibliographical Pedagogy ${ }^{1}$
}

\section{Introduction}

Once highlighted as a hobbyist's novelty, allied technologies such as 3D scanning, 3D modeling, and 3D printing are fueling vital new advances in a diversity of fields: in biomedical research, human tissue is being $3 \mathrm{D}$ printed to form human organs; the development of $3 \mathrm{D}$ printed titanium parts in aerospace engineering will save airplane manufacturers millions of dollars per plane; and 3D printing allows mathematicians to create intricate physical representations of geometric models that are otherwise difficult to visualize. ${ }^{2}$ Though 3D technologies have only recently gained a similar foothold throughout the humanities, the results are no less encouraging. Perhaps unsurprisingly, these projects focus in large part upon furthering our understanding of physical artifacts. Recent examples range from detailed replicas of delicate fossils and high-resolution 3D scans of engraved wooden blocks to 3D models of damaged paintings and fragile medieval artifacts. ${ }^{3}$ Application of these technologies naturally aligns with the study of material culture, facilitating the understanding of and access to rare and delicate materials. The appearance of 3D technologies within special collections is thus a fitting development.

Within our field, these technologies have the potential to greatly expand the pos-

1. Aspects of the project described in this paper have been presented at the Texas Conference on Digital Libraries (2017) and the annual conference of the ALA Rare Books and Manuscripts Section (2017).

2. Lydialyle Gibson, "Building Toward a Kidney,” Harvard Magazine, Jan.-Feb. 2017, 37-43; Henry Fountain, "At the Printer, Living Tissue," New York Times, Aug. 18, 2013; James Vincent, "3D-Printed Titanium Parts Could Save Boeing up to \$3 Million per Plane," The Verge, Apr. 11, 2017, available online at https: / www.theverge.com/2017/4/11/15256008/3d-printed-titanium-parts-boeing-dreamliner-787 [accessed 1 February 2018]; Luke Whelan, "Can't Imagine Shapes in 4 Dimensions? Just Print Them Out," Wired, Nov. 15, 2016, available online at https://www.wired.com/2016/11/ cant-imagine-shapes4-dimensions-just-print [accessed 1 February 2018].

3. Sophie Bushwick, "3-D Printing Gets Ahead: Anthropologists Use Printing Technology to Model Fossils," Scientific American, Sept. 19, 2011, available online at www.scientificamerican.com/article/ three-3d-printing-anthropologists-use-printing-technology-to-model-fossils [accessed 1 February 2018]; Michael Hancher, "Imaging Wood Engravings," available online at https: / / storify.com/MichaelHancher/imaging-wood-engravings [accessed 22 February 2018]; Xavier Aure, Paul O’Dowd, and Joseph Padefield, "Generating 3D Models of Paintings Through the Combination of 2D, 3D and RTI Data," in Proceedings of the Conference on Electronic Visualization and the Arts, eds. Jonathan Bowen, Nick Lambert, and Graham Diprose, 25-32.

๑ 2018 by Courtney Jacobs, Marcia McIntosh, and Kevin M. O'Sullivan (CC BY-NC [https://creativecommons.org/licenses/by-nc/4.0/]). 
sibilities of how we engage students and researchers in hands-on outreach and instruction. In the past ten years, we have seen a flood of inexpensive, user-friendly, tabletop 3D printers, rendering this once cost-prohibitive technology accessible to entry-level consumers. Increasingly, librarians are providing access to these innovative resources, incorporating maker culture into their growing list of services. With this access, the special collections community now has the opportunity to develop high-quality teaching models to expand learning and outreach. One area where this is particularly true is book history instruction. Special collections librarians are often hampered in their attempts to prepare effective curricula by the increasing cost of acquiring teaching tools from the hand-press era. However, the growing makerspace movement may provide a lasting solution to this persistent problem. The 3Dhotbed project (an acronym for "3D Printed History of the Book Education”) harnesses maker culture to advance book history instruction by providing open-access 3D data through a digital library platform. This collaboration was developed to provide $3 \mathrm{D}$ printable teaching models of tools used in book production during the hand-press period based upon working facsimiles in use at Texas A\&M University's Book History Workshop. ${ }^{4}$

\section{The Case for Incorporating 3D Technologies into Special Collections}

For the majority of librarians and faculty teaching book history, a lack of periodspecific artifacts requires instructors to rely on readings, diagrams, and videos of the tools and processes that enabled the print revolution of the fifteenth century. Without the assistance of these artifacts as physical aids, providing detailed instruction on the related mechanical and technological innovations of early printing can lead to instruction that remains opaque or convoluted. To augment this curriculum, many institutions struggle to amass a teaching library of book history artifacts, a process that is cost-prohibitive as well as time- and labor-intensive.

The need for robust, hands-on bibliographical instruction is well documented. As early as the turn of the twentieth century, eminent scholars such as Ronald B. McKerrow called for a student to experience "all the processes through which the matter of the work before them has passed, from its being written down by the pen of its author to its appearance in the finished volume." ${ }^{{ }_{5}}$ Decades later, the Book History Workshop at Texas A\&M University was formed to answer this need, developing a process-driven, experiential approach to the analysis of rare

4. More information is available at http://3dhotbed.info [accessed 2 April 2018]. The data are available in the University of North Texas Digital Library at https:// digital.library.unt.edu/explore/collections/THREED/ [accessed 2 April 2018].

5. Ronald B. McKerrow, "Notes on Bibliographical Evidence for Literary Students and Editors of English Works of the Sixteenth and Seventeenth Centuries," Transactions of the Bibliographical Society 12 (1911-13): 220. 
materials, dubbed "Empirical Bibliography."' Over the course of several years, the workshop has continued to expand, incorporating additional related learning modules such as paper marbling and punch cutting. However, practical problems associated with "acquiring the necessary range of historical equipment," which "inevitably demand[s] expenditures of time and resources" persist." This, it seems, has historically been the largest hurdle for instructors who seek to engage students in experiential bibliographical exercises.

To source the equipment used in the Book History Workshop, special collections librarian Steven Escar Smith partnered with Stephen Pratt, a craftsman and artisan who had prior experience creating historical reconstructions. ${ }^{8}$ These tools were painstakingly researched and handcrafted and have proven indispensable in support of the workshop instruction. Among the equipment Pratt built were an English common press, several smaller parlor presses, dozens of wooden composing sticks, and two adjustable hand molds. The latter are used in conjunction with a selection of punches and a complete set of matrices to teach the punch matrix system ${ }^{9}$ during the workshop. To learn this process, students use the punches to strike a matrix for a single letterform; this is inserted into the hand mold from which is cast a piece of type using lead alloy. For the students, handling the physical tools for this laborious, multistep process is crucial to understanding the rudiments of hand-press type foundry and typography, and other related topics in book history. Without these tools, it would be nearly impossible to meaningfully articulate the nuances of casting type with a hand mold and why these techniques were so crucial to its success as a device.

The working replicas that Pratt created have now been put to a new use as the exemplars upon which the 3D printable teaching tools are based. In partnership with the Book History Workshop, the 3Dhotbed project increases the impact and potential reach of these bespoke teaching implements and removes a barrier to effective book history pedagogy. For the first time, the data necessary to $3 \mathrm{D}$ print high-quality

6. Todd Samuelson and Christopher L. Morrow, "Empirical Bibliography: A Decade of Book History at Texas A\&M," Papers of the Bibliographical Society of America 109, no. 1 (2015): 83.

7. Ibid., 84.

8. Ibid., 94 .

9. The invention of the punch matrix system for typecasting, often credited to Johannes Gutenberg around 1450, brought on the advent of moveable metal type in Europe. A hand mold is a 2-part tool for casting type; slid together, the two parts of the mold create a cavity that adjusts for varying letter widths. The type design process begins with a punch, a long steel bar with a single character carved in relief on the long end. A type designer carves a punch for each character necessary for printing (uppercase and lowercase letters, punctuation marks, and so on). The completed punch is used to strike a matrix: a small rectangular block of copper onto which the character is incised via the punch. The typecaster inserts a matrix for a specific letter into the hand mold and fills the resulting cavity with molten type metal, creating an individual piece of type, or sort. This process is repeated until the desired number of sorts have been cast. The typecaster inserts a new matrix for another character into the mold and continues casting type until an entire font has been cast. 
models are available at no cost to institutions engaged in this curriculum. While these models cannot be used to cast type using molten lead alloy, the complete toolkit allows the student to follow the iterative processes involved in type design and typecasting in greater detail than has even before been practically available.

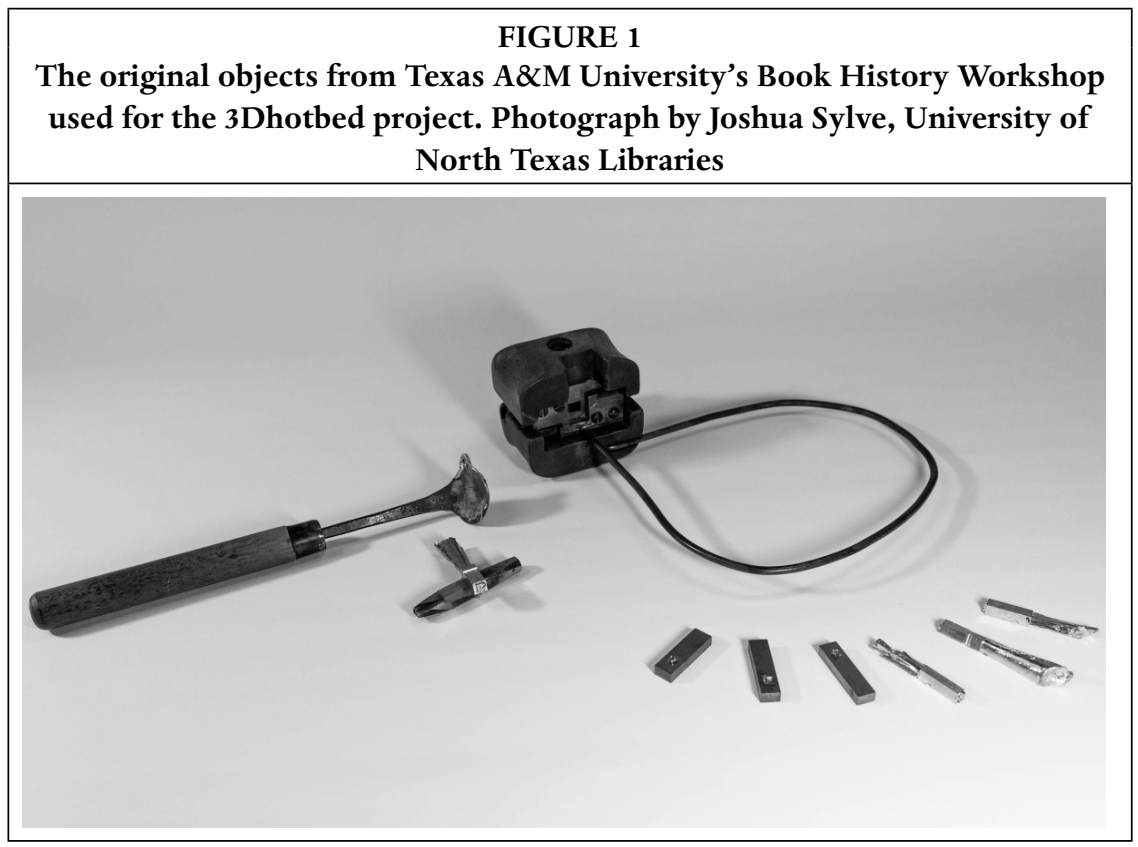

In 2016 and 2017, the 3Dhotbed group created a complete set of the tools used in early typecasting by using current rapid fabrication technologies and engaging in multiple aspects of maker culture, thus positioning the librarian as both the consumer and the maker. First, high-quality 3D scans of the teaching tools were produced. The resulting data sets were uploaded to an institutional repository, ensuring the public's free and open access to these data. These data sets provide an opportunity for tool fabrication to persons interested in furthering their own book history instruction programs, thus enabling institutions to use their community's makerspaces to expand pedagogy.

\section{Dhotbed: A Test Case for the Use of 3D Technologies within Special Collections}

In developing the teaching toolkit, the 3Dhotbed project employed 3D technologies that are state-of-the-art but also commonly held by university makerspaces, thereby testing the potential for academic librarians across the field to endeavor upon similar projects at their own institutions. Using the current technology, there are two fundamental approaches to creating a 3D model of an object: hand modeling and 3D scanning. In hand modeling, a model is designed from scratch 
using software. ${ }^{10}$ Models can also be created through one of the many $3 \mathrm{D}$ scanning methods to capture the precise measurements of the original object. In practice, the two approaches are often used simultaneously. The 3Dhotbed project relied on both laser scanning and hand modeling to create 3D printable models of the desired teaching tools.

The team initially planned to scan and print exact replicas of the multiple tools provided by the Book History Workshop using primarily technology and makerspace services available in-house. However, ensuring a functional set of tools eventually required contracting with external vendors when project participants' budding skills could not achieve the desired output. Initial scanning was conducted in-house at the University of North Texas Digital Projects Lab, as well as some postprocessing. For additional scanning, hand modeling, and adaption of the smaller pieces to the final hand mold, the group sought assistance from outside vendors.

Two hand molds, a punch, a matrix, and a piece of cast type with the jet ${ }^{11}$ attached were identified for inclusion in the teaching toolkit. The punch identified for the project (and thus the resulting matrix and type pieces) was for a majuscule blackletter B. Using a NextEngine HD 3D scanner, the team scanned the smaller of the two hand molds. Initial scans necessitated various pretreatments due to the reflective nature of the materials. The mold was powdered heavily with talc to reduce reflection and create cleaner scans, which were then further cleaned, aligned, and postprocessed. The resulting model was exported into an STL file. Since the overall goal was to produce a model that could be used to demonstrate typecasting during instruction, the team printed a miniature prototype of the smaller mold to ensure both sides would fit together snugly. This half-scale mockup was soon followed by full-size prototypes. The first beta prints provided a proof-of-possibility and started what would be an iterative process of beta-printing and model refining. Several $\mathrm{ABS}^{12}$ filament models were printed in the University of North Texas Libraries' makerspace, The Factory, and with local vendors identified through an online portal for accessing freelance 3D printing services, 3Dhubs.

After scanning and printing prototypes of both the larger and smaller hand molds, the team identified the former as ideal for the final printed set, as it had been used

10. This technique has been in practice since the technology's earliest beginnings and is among the most popular. Examples of commonly used modeling software include Blender, Meshlab, or Meshmixer. The designer molds and crafts a collection of basic shapes supplied by the software to create the model of their choosing, using his or her imagination or extant physical object as reference.

11. When casting type by hand, the resulting type piece includes an attached piece of waste metal, known as a jet, that is removed before the type is cleaned and distributed.

12. Acrylonitrile butadiene styrene, or ABS, is one of the two most common filaments used in $3 \mathrm{D}$ printers using FDM (fused deposition modeling) technology. The other, PLA (polylactic acid) is a biodegradable biopolymer made from renewable materials. Both filaments are thermoplastics; they become malleable when heated. 
to cast the pieces of type that were scanned to be included with the pedagogical toolkit. Doing so ensured that the complete set of models would fit together. However, the larger mold included a spring that proved difficult to scan, given the NextEngine's limited field of view. ${ }^{13}$ In an attempt at a workaround, the team again consulted outside expertise. Using a handheld Ein Scan Pro 3D scanner, the contractor was able to scan the complete mold with spring. However, including the spring in the final STL file nearly doubled the model's printing time and amount of requisite filament, as it necessitated a large support structure for printing. Moreover, the spring on the resulting printed model was fragile and prone to breakage during the cleaning process. Since this spring's function on the original artifact was to hold the matrix in place during casting, and a 3D printed facsimile spring would lack the properties necessary to replicate that function, the team decided to excise it from future iterations. Additional modeling reduced stickiness, allowing the two parts of the mold to slide together easily.

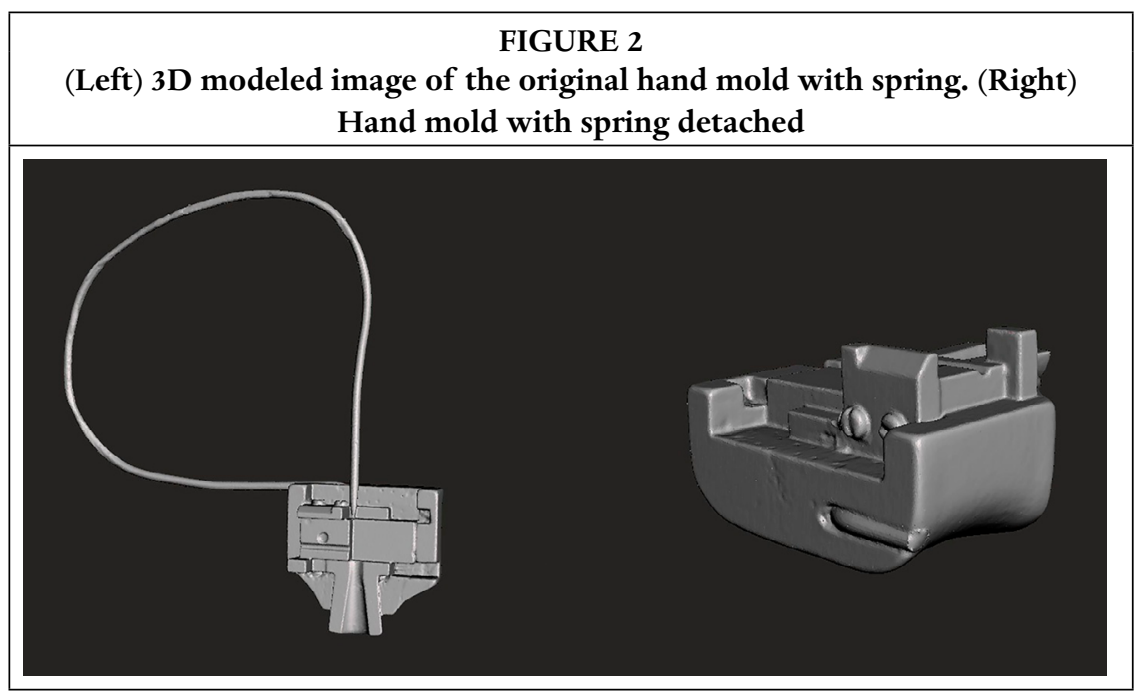

With complete data sets and prototypes for the two-part hand mold finalized, the group sought to scan and create the smaller parts of the teaching set: the punch, matrix, and piece of type. Like the metal portions of the hand mold, these artifacts made from metallic alloys required pretreatment to obtain functional scans. Powdering did not yield better results, so the team experimented by applying matte white spray paint to the shinier type pieces. While this improved the physical capture of the item, the finer details of the letterform itself remained elusive to the scanner.

13. In practice, this large metal spring, affixed to the top part of the hand mold, was used during typecasting to hold the matrix in the mold and increase the speed at which type was cast. Depending on the mold, this spring can more than double the total height of the mold. 
Multiple outside vendors suggested that hand-modeling these pieces using the existing object scans for size and measurement references would produce cleaner data than printing directly from source scans. One of the project's key objectives was to provide an educator anywhere in the world the ability to reliably print the 3 Dhotbed model, so it was paramount that the STL data, and its resulting printed model's detail, be as clear as possible. To that end, the team decided to partner with a local vendor who provided further assistance improving the final dataset. After comparing multiple vendors' fee structures for modeling, the group identified a vendor who was able to most efficiently produce clean outlines of the object shapes. Instead of recreating a true facsimile of the physical artifacts with the original type, the vendor used a modern, sans-serif majuscule B. The vendor also modeled a detachable jet piece that better illustrated this step in the typecasting process. Last, he extrapolated the resulting data into the hand mold design to ensure that all parts would fit together once printed.

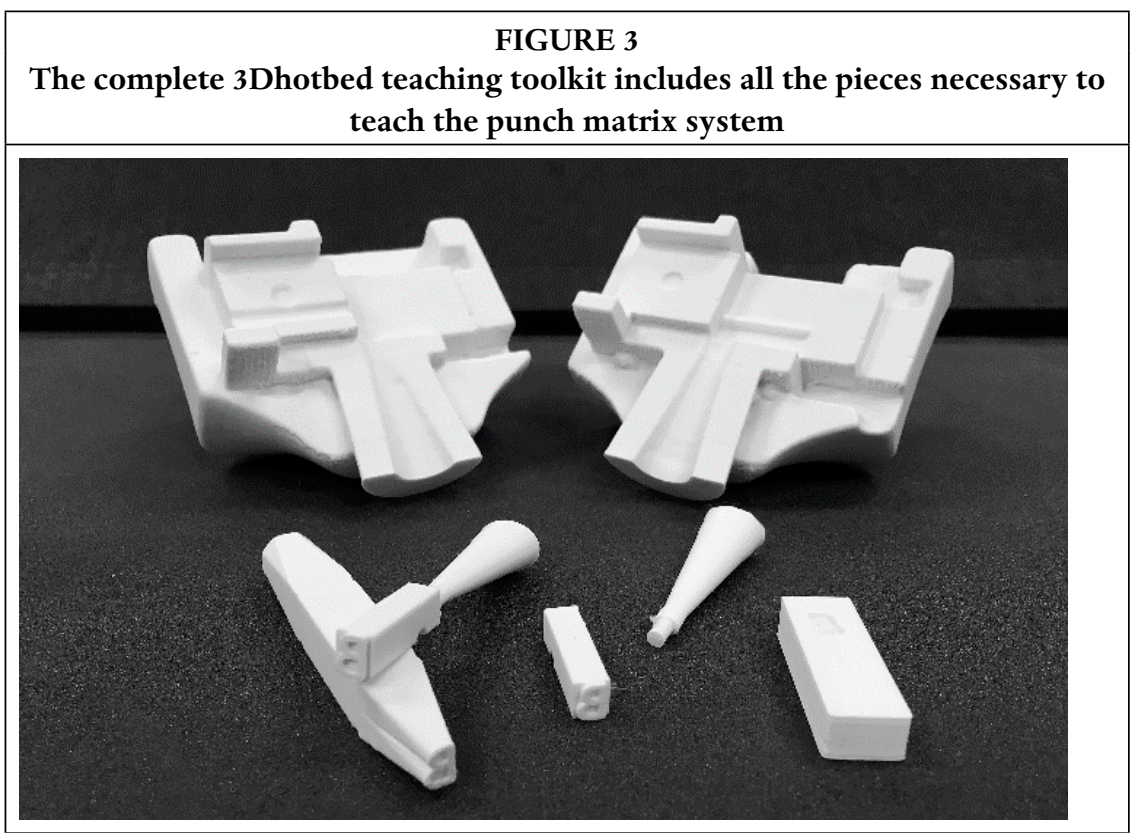

The first prototype models were printed using ABS. The final set and other models printed by the outside vendor used PLA. These filament types, being among the most common, were used in the hopes that the set could be replicated by anyone. The 3Dhotbed team plans to experiment with exotic filaments and other 3D printing processes in the future. For example, iron-based PLA could produce a set of magnetizable models, increasing ease of use during instruction. Certain pieces would benefit from the increased detail possible with resin-printing, though this process remains somewhat cost-prohibitive. A high-quality, detailed resin print 
could eventually open the possibility for end-users to employ investment casting to manufacture a functional metal hand mold. ${ }^{14}$

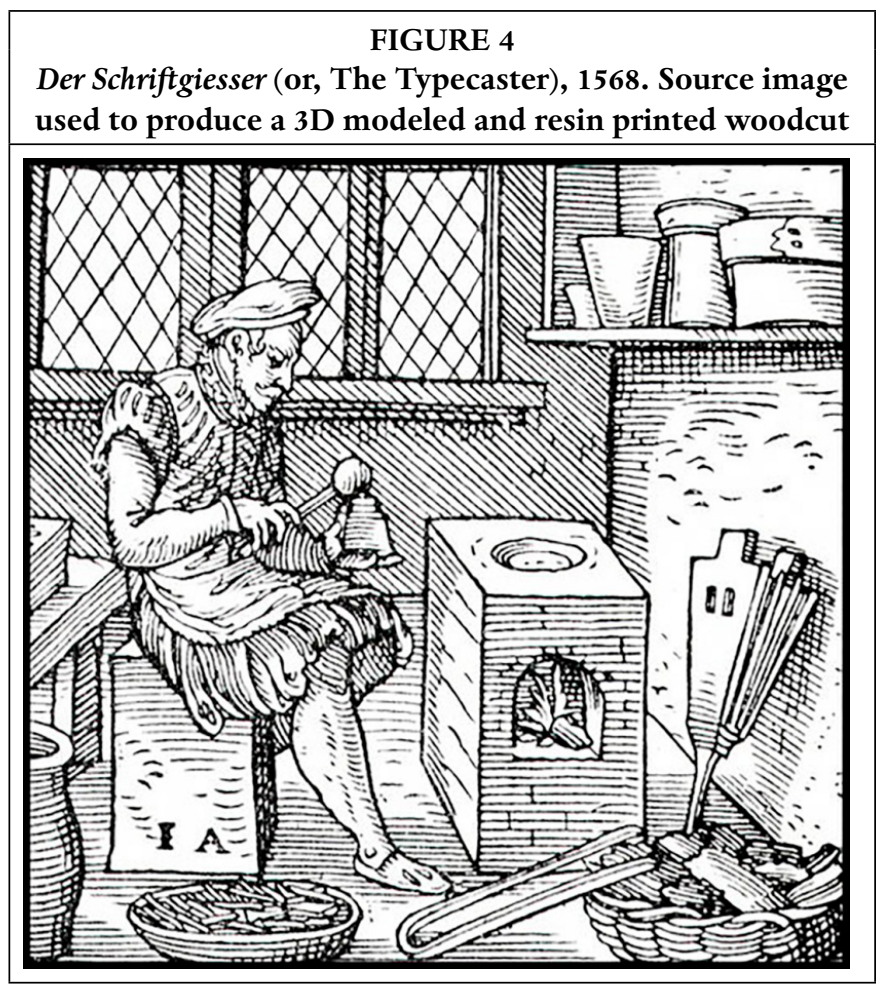

Forging relationships with outside vendors contributed significantly to the project's success by decreasing prototype development time and expediting the speed at which the toolkit was completed. These relationships also facilitated discovery and creation. In discussing the steps required to hand-model the smaller parts of the toolkit, the team recognized an opportunity to fabricate other items commonly used in book history education even when the team did not have access to the physical objects. Since the process of hand-modeling allows the creator to extrude any design out of a flat surface, the team was able to easily create a facsimile woodcut block from a source image. This process produced much cleaner results than were possible by $3 \mathrm{D}$ scanning an actual woodcut, and did not require access to the original—in this case, a woodcut for "Der Schriftgiesser" (The Typecaster), a woodcut illustration from Sachs and Amman's sixteenth-century text, often referred to as Das Ständebuch, or The Book of Trades. ${ }^{15}$ Using a 2D scan of the black-

14. Investment casting is a manufacturing process that uses a wax model of an object as a reverse mold to replicate that object in solid metal. The object is coated with refractory ceramic material, which conforms to the shape of the model being cast, and the model is then melted out of the hardened cast. The resulting cavity is filled with molten metal and broken out of the cast.

15. Hans Sachs and Jost Amman. Eygentliche Beschreibung aller Stände auff Erden, hoher und nidriger, geistlicher und weltlicher, aller Künsten, Handwercken und Händeln (Frankfurt am Main: Feyerabend, 1568), 20. 
and-white image, the vendor extruded the black lines of the image from a block using modeling software, setting the height of the completed dataset at type-high, 0.918 inches. Once printed, the resulting data set produced a 3D facsimile that accurately reproduced the original image and that can be locked in a form on the bed of a printing press. Unlike zinc or polymer plates, which replicate only the printed surface area, the resin-printed facsimile reproduces the entire object, thus making a more faithful teaching tool for demonstrating relief book illustration.

While the benefits of partnering with outside vendors are numerous, it is important to note that doing so can be costly. Incorporating external assistance for modeling and printing should be scrutinized as part of cost-benefit analyses throughout the project. In identifying vendors, the team found that cost structures varied widely between institutions. ${ }^{16}$ The team ultimately chose to work with a local vendor who did not charge for prototyping and allowed pickup in person (thus eliminating shipping fees). Additionally, certain 3D printing technologies are not yet as ubiquitous and therefore remain cost-prohibitive to many. While the 3Dhotbed teaching toolkit is easily produced on a standard FDM printer, the increased detail of the woodblock facsimile was only possible through stereolithography, or resin printing. ${ }^{17}$ Depending on the institution's makerspace services and fees, it is possible to replicate the 3Dhotbed project, from scanning and modeling to final prints, within a library makerspace, and the team welcomes comments and suggestions from others wishing to engage in similar projects.

\section{Lessons Learned in Extending Bibliographical Instruction through 3D Technologies}

The first phase of the 3 Dhotbed project, completed in the spring of 2017, designed and manufactured a complete teaching toolkit, a discrete set of models that are intended to function as an instruction unit for teaching the punch matrix system. Using the toolkit, one can demonstrate how type was cast with an adjustable hand mold, a punch, a matrix, and a piece of cast type (complete with a removable jet). The data to print additional tools are also available, such as the woodblock facsimile as well as a composing stick into which the type may be set.

During the project's testing phase, the 3Dhotbed teaching toolkit was employed in special collections classroom instruction at the University of North Texas,

16. Some makerspaces charge only for materials, other by the hour. Some makerspaces require users to complete training courses prior to using technology; other labs act as services wherein files are submitted and knowledgeable technicians complete all necessary printing work. Certain makerspaces are staffed with individuals trained to assist with prototyping and problem-solving; others are completely DIY.

17. Fused Deposition Modeling (FDM) printers heat a thermoplastic filament to its melting point and extrude it, layer by layer, to create a three dimensional object. In stereolithography (or resin printing), photosensitive liquid resin is exposed to certain light frequencies, hardening layer by layer, fabricating an object with increased detail on a smaller scale. 
augmenting the curriculum of courses taught regularly throughout the semester. Librarians partnered with teaching faculty to develop multiple lectures and class visits where students benefited from hands-on experience analyzing and describing primary materials. For a graduate-level methods course in the English department, librarians and teaching faculty integrated both collections materials and book history instruction into course curricula. The 3 Dhotbed toolkit was used alongside other teaching tools, such as bibliographic format sheets, a loose assemblage of machine-made type and linotype slugs, and composing sticks to introduce graduate students to the basics of analytical and descriptive bibliography.

Although students studied their assigned course readings and watched relevant videos in advance, many had questions related to the rudimentary processes of designing and casting type, thus indicating that these strategies alone were not effective in describing the individual steps that would together produce a single piece of type. In particular, the students struggled with understanding the difference between typecasting (the formation of a piece of type from hot lead alloy using an adjustable hand mold) and type design (the series of processes, including cutting a punch and striking a matrix, that lead up to the casting process). To elucidate these practices, the toolkit was used to provide hands-on illustration of these concepts. Using the 3Dhotbed toolkit, each student was able to handle the tools and formulate his or her own understanding of the principles via an experiential learning exercise-an opportunity not afforded through readings and instructional videos. Through nesting the punch and matrix together, students saw how one begets the other in the design of a letterform. Likewise, by then setting the matrix within the hand mold and positioning the sample piece of type, students understood the relationship between the two processes and, furthermore, how a piece of type was produced.

By successfully employing the 3Dhotbed toolkit in book history curriculum, the group has demonstrated that the processes of three-dimensional scanning and additive manufacturing provide the ideal technology not only to produce difficultto-source teaching tools, but (perhaps more significantly) supply an affordable means for doing so. Now these tools are available for the wider public to download and print themselves. While the data are free, the investigators involved with the project encourage and look forward to feedback regarding instructors' success in producing the tools, how they incorporate the toolkit into their curricula, and any suggestions for other objects from the annals of book history they would like to see added to future toolkits.

Upon developing the complete teaching toolkit, the group held a Book History Maker Fair event to showcase the project's progress and solicit feedback from participants outside the traditional special collections curriculum setting. Here, 
librarians, makers, and letterpress printers all convened to demonstrate multiple aspects of book history. At each station of the event, participants replicated historical processes from the hand-press period to create their own informative pamphlet. Using the 3Dhotbed toolkit, they learned how type was designed and cast by hand. Another station allowed attendees the opportunity to set individual pieces of metal type using a composing stick. At another station, event participants printed a cover binding for their pamphlet on a tabletop letterpress set with the resin-printed sixteenth-century woodcut facsimile. Using this cover, they folded and stab-sewed a quarto pamphlet containing a glossary of book history terms to take home. Alongside these hands-on stations, participants interacted with examples of rare books, early iterations of the toolkit's components, and various models of 3D printers from the University of North Texas's makerspace, The Factory, thus bridging more than half a millennium in the development of printing.

\section{The Bigger Picture (Extending It Further)}

The 3Dhotbed project began as a collaboration with an initial purpose: to create a $3 \mathrm{D}$ printed facsimile of an adjustable hand mold and related tools used in typecasting for use in an instructional setting. Beyond this initial success, a key takeaway from the project's early phases was the far-reaching potential of incorporating $3 \mathrm{D}$ technologies into not only book history instruction, but special collections more generally. The popularity of the 3Dhotbed teaching toolkit, and the support afforded by the ability to ingest 3D data sets into a publicly available digital repository, paves the way for additional collaborations to expand access to these rare and often fragile aspects of material culture. In the future, libraries will build publicly accessible virtual repositories of their physical holdings, which will be available for scholars, instructors, or the public to investigate and perhaps download and 3D print. Indeed, the early stages of this work is already taking place, as $3 \mathrm{D}$ technologies make their first appearances in special collections and museum institutions.

The data produced by these efforts will have the potential to radically change the way our profession envisions learning and outreach. The rapid advancements in 3D technologies we are currently witnessing suggest that these collaborations could go further than creating facsimiles of rare and fragile items. With the parallel rise of virtual reality technology, one can imagine a day when digital instruction sessions incorporate high-quality 3D scans of these objects, allowing students to log in from anywhere in the world and virtually experience the process of casting type using an adjustable hand mold or working an English common press. We are already seeing that today's technological advancements will provide our field multiple opportunities to integrate collection materials in new and more meaningful ways. More important, these tools will allow us to connect users with collections at a larger scale regardless of geographical location or institutional affiliation. 